\title{
The impact of non-caloric sweeteners on male fertility: a systematic review in rodent models
}

\author{
M. Kearns ${ }^{1,2}$, F. MacAindriu ${ }^{1}$ and C. Reynolds ${ }^{1,2}$ \\ ${ }^{1}$ School of Public Health, Physiotherapy and Sports Science, University College Dublin, Dublin, Ireland and \\ ${ }^{2}$ Conway Institute /Institute of Food and Health, University College Dublin, Dublin, Ireland
}

Consumption of non-caloric sweeteners (NCS) has increased in recent years and are a popular sugar substitute, providing intense sweetness with minimal or no energy ${ }^{(1)}$. NCS are abundant in our food supply and found in consumable products such as diet beverages, yoghurts and chewing gum ${ }^{(2)}$. Studies in rodents have shown adverse effects, including increased oxidative stress ${ }^{(3)}$ and elevated glucose concentrations ${ }^{(4)}$. Despite noted effects on metabolic outcomes. there is limited research on the impact of NCS on human reproductive health, with some evidence of in utero exposure to NCS promoting pregnancy complications ${ }^{(5)}$. Female rodent studies has found associations between NCS consumption and reduced pregnancy length, with adverse outcomes reported in offspring, including reduced fetal growth ${ }^{(6)}$, however the effect of NCS on reproduction has been relatively under-researched in a human setting, particularly in relation to male reproductive outcomes. We therefore set out to systematically examine the literature in relation to pre-clinical rodent studies investigating the role of NCS on male fertility outcomes.

A literature search was performed in Pubmed, Embase, Web of Science and Scopus to find all studies investigating the effects of NCS on male fertility when compared with controls or a nutritive sweetener group. Searches were conducted for the following NCS; aspartame, stevia, acesulfame k, advantame, saccharin, neotame, cyclamate and sucralose. Primary outcomes were effects on sperm quality, quantity, or pregnancy success. Secondary outcomes were effects on reproductive organ weights, litter size and gestational length. Two reviewers independently screened studies for inclusion and carried out a risk of bias assessment in Covidence, using the Syrcle risk of bias tool.

In total, 9 studies met our inclusion criteria and were included in our review. The NCS studied in mice and included in the final studies were aspartame, advantame, rebaudioside a (stevia), saccharin, and sucralose. Acesulfame k, neotame and cyclamate did not arise in our searches and were not included. Overall, $44 \%$ of studies showed a negative effect of NCS on male reproductive parameters, with an observed reduction in sperm count, compared with controls. $33 \%$ of the studies observed significant reductions in sperm motility, viability, and an increase in abnormal sperm in the NCS group, however only one included study on saccharin used the appropriate ADI equivalent to humans of $<5 \mathrm{mg} / \mathrm{kg}$ bw/day, while the remaining NCS exceeded the ADI for human consumption. Two studies showed a dose-dependent effect of NCS on fertility parameters and four studies reported decreased reproductive organ weights. Studies using advantame and sucralose observed no effect on male fertility, however in the advantame study, only high dose groups were assessed and in the sucralose study, sperm motility and viability were not assessed.

NCS seem to affect male fertility in rodents, with some studies showing significant effects on reduced sperm quality and quantity in rodents consuming aspartame, saccharin, and stevia. There may be a dose-dependent manner of NCS effects, with higher intakes associated with worse outcomes, compared with lower intakes and controls. The specific effects of NCS on fertility have been conflicting and the available studies have been heterogeneous in terms of the study duration, sample size and the doses evaluated, with most studies using NCS doses well above the ADI for humans. Further research in humans is needed to assess whether NCS could be a potential cause of male fertility issues.

\section{References}

1. Sharma A, Amarnath S, Thulasimani M, et al. (2016) Indian J Pharmacol 48(3), 237-40.

2. Gardner C, Wylie-Rosett J, Gidding SS, et al. (2012) Diabetes Care 35(8), 1798-808.

3. Mourad IM (2011) African J Pharm Pharmacol 5(6), 678-82.

4. Palmnäs MSA, Cowan TE, Bomhof MR, et al. (2014) PLoS One 9(10).

5. Englund-Ögge L, Brantsæter AL, Haugen M, et al. (2012) Am J Clin Nutr 96(3), 552-9. 